\title{
Multi-Dimensional Consequentialism and Risk
}

\author{
Vuko Andrić ${ }^{1}$ - Attila Tanyi ${ }^{2}$
}

Accepted: 31 October 2015 /Published online: 12 December 2015

(C) The Author(s) 2015. This article is published with open access at Springerlink.com

\begin{abstract}
In his new book, The Dimensions of Consequentialism, Martin Peterson proposes a version of multi-dimensional consequentialism according to which risk is one among several dimensions. We argue that Peterson's treatment of risk is unsatisfactory. More precisely, we discuss a number of major problems of one-dimensional (objective or subjective) consequentialism, and show that none of them disappears with Peterson's proposal. In ending our paper, we address the objection that our discussion overlooks the fact that Peterson's proposal is not the best version of multi-dimensional consequentialism. Our reply is that the possibilities of improving multi-dimensional consequentialism are very limited as far as risk is concerned.
\end{abstract}

Keywords Consequentialism $\cdot$ Moral aspect $\cdot$ Moral dimension $\cdot$ Risk $\cdot$ Martin Peterson

\section{Introduction}

A long-drawn controversy in normative ethics among advocates of consequentialism concerns the deontic status of risky acts. There are two standard positions:

Objective consequentialism (OC): an act is right if and only if no alternative would have better consequences; otherwise the act is wrong.

Subjective consequentialism (SC): an act is right if and only if no alternative has larger expected value; otherwise the act is wrong. ${ }^{1}$

\footnotetext{
${ }^{1}$ There are, of course, more (albeit less prominent) positions concerning the relevance of risk for rightness (e.g., relativism and contextualism), which we ignore in order to keep things simple.

Attila Tanyi

Attila.Tanyi@liverpool.ac.uk

Vuko Andrić

vuko_andric@yahoo.de

1 Universität Mannheim, 68131 Mannheim, Germany

2 Department of Philosophy, University of Liverpool, 7 Abercromby Square, Liverpool L69 7WY, UK
} 
According to OC risk does not affect the deontic status of acts, whereas it does - by affecting the expected values of acts - according to SC. To illustrate the difference between the positions, take the following example: Experts disagree about what needs to be done in order to prevent climate change. Act $A$ is the strategy of doing nothing and could result in a catastrophe. Act $B$ consists in spending large sums of money on mitigating measures. Act $C$ is the most costly strategy but also delivers a dramatic reduction of the emission of greenhouse gases. ${ }^{2}$ The example is represented in Table 1 (the numbers in the table represent moral value or, as we can assume for simplicity, well-being).

If $s$ will turn out to be the actual state, then OC implies that $A$ is right and $B$ and $C$ are wrong. If $t$ is the actual state, then $\mathrm{OC}$ implies that $B$ is right and $A$ and $C$ are wrong. SC, on the other hand, makes the analysis depend on risk, i.e. the probabilities of states $s$ and $t ; p(s)$ and $p(t)$. In particular, the rightness of an act is given by the expected value of the various acts.

In his book The Dimensions of Consequentialism, Martin Peterson argues that he can offer an alternative theory, a version of what he calls multi-dimensional consequentialism (MDC), that "tallies better with our considered moral intuitions" than both OC and SC (101). ${ }^{3}$ To understand Peterson's proposal about the connection between risk and consequentialism (which is a specific version of MDC), we first have to grasp the essentials of MDC. The defining thesis of MDC is that the deontic status of an act is determined by several moral aspects that belong to irreducible moral dimensions (3). However, Peterson thinks that we need to add two further claims to MDC.

The first claim is that "[t]he binary relation 'at least as good consequences as' is not a complete ordering." (9) The idea behind this thesis is that the different moral aspects are either incomparable or on a par. How widespread this phenomenon is, i.e., whether all or most or only some moral aspects come under the scope of the first additional claim, is left unclear by Peterson.

The last defining thesis of MDC is that moral rightness and wrongness come in degrees: they are non-binary entities (10). Peterson's idea is simple: some acts are partly right and partly wrong. He brings as intuitive example a case where you can bring about either a world in which Alice gets 100 units of well-being and Bob gets 50, or a world in which Alice and Bob get 60 units each (2). The first option, Peterson says, is right with respect to well-being as it brings about more well-being than the second. The other option is right with respect to equality. All things considered, no option is entirely right or wrong.

What does Peterson say about risk? The most important point is that he treats risk as one crucial moral dimension, along with well-being and equality. Let us go back to Table 1 . If the probability of state $s$ is $p$ and the probability of state $t$ is $1-p$, then according to Peterson's Proposal (PP) - i.e. the version of MDC Peterson endorses - the respective degrees of rightness in the risk dimension of $A$ and $B$ are $p$ and $1-p$ (105). Hence we get a modified table - Table 2.

\footnotetext{
2 The example is borrowed from Peterson (2013: 102).

${ }^{3}$ Page references, unless otherwise stated, are to Peterson (2013) throughout the paper. While we talk about OC and SC, Peterson talks about "actualism" and "possibilism". Peterson uses the label actualism to refer to the position "that only actual consequences matter" (100), whereas possibilism is a name for the position "that an act's deontic status depends on what could happen, not merely on what actually happened" (101). His chapter is de facto concerned with what we call objective and subjective consequentialism and others call actual-value and expected-value consequentialism. This is clear from the arguments he considers and from the writings he mentions.
} 
Table 1 OC, SC, and risk

\begin{tabular}{lll}
\hline & State $s$ & State $t$ \\
\hline Act $A$ & 100 & -100 \\
Act $B$ & -10 & 10 \\
Act $C$ & 1 & 1 \\
\hline
\end{tabular}

So according to PP (in contrast to SC), it is only having the best consequences that tends to make acts right with respect to risk (107). At the same time, PP holds (in contrast to OC) that in cases of risk an act's probability of having the best consequences figures in its deontic status (108). Once PP is accepted, it is fed into the general framework of MDC. That is, along with the other moral aspects - well-being and equality - it is used to give an all-things-considered moral verdict as to the (degree of) rightness (wrongness) of a particular act.

\subsection{The problems of Peterson's Proposal}

This is then the proposal that is claimed to be more in line with our intuitions than either OC or SC. Now, the intuitive appeal of these positions is typically tested by how well they respond to certain problems raised by their opponents. Here is a list of important problems:
a) The Jackson case
b) Action-guidance
c) 'Ought' implies 'can'
d) Decisiveness of optimal consequences
e) Normative disagreement

Of these, a) to c) pose challenges to OC, d) and e) to SC. Peterson himself discusses only a) and d), but given his claim, it is warranted to investigate the intuitiveness of MDC in response to the other problems as well. Our strategy in this regard will be simple. We will show that all problems of OC and SC are problems for PP as well. ${ }^{4}$

\section{a) The Jackson Case}

We begin with a case that was put forward by Frank Jackson. The original description reads:

\section{The Jackson case.}

"Jill is a physician who has to decide on the correct treatment for her patient, John, who has a minor but not trivial skin complaint. She has three drugs to choose from: drug A, drug B, and drug C. Careful consideration of the literature has led her to the following opinions. Drug A is very likely to relieve the condition but will not completely cure it.

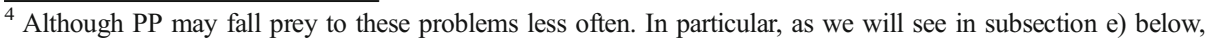
there seem to be cases of normative disagreement PP can make better sense of than SC. Note also that, admittedly, the list of problems above is not exhaustive. Hence our claim is justified by induction and one might rebut it by showing that there is at least one problem of OC or SC, respectively, which is not a problem for PP. However, we do not know of such a problem; moreover, it would still be a blow to PP if it turned out that PP is haunted by all the problems listed above.
} 
Table 2 MDC and risk

\begin{tabular}{llll}
\hline & State $s$ & State $t$ & Degree of rightness \\
\hline Act $A$ & 100 & -100 & $p(s)$ \\
Act $B$ & -10 & 10 & $p(t)$ \\
Act $C$ & 1 & 1 & 0 \\
\hline
\end{tabular}

One of the drugs $\mathrm{B}$ and $\mathrm{C}$ will completely cure the skin condition; the other though will kill the patient; and there is no way that she can tell which of the two is the perfect cure and which the killer drug. What should Jill do?" (Jackson 1991: 462-3)

In his own discussion of the case, Peterson rightly points out that Jackson uses the example as a "plea for risk aversion": the intuition the case is designed to reveal is that prescribing drug $A$ is all-things-considered right and prescribing drug $B$ or drug $C$ is all-things-considered wrong. This clearly contradicts OC.

We do not consider possible responses proponents of OC might have to the Jackson case, but point out that if the Jackson case poses a problem for OC, then PP shares this problem. The reason is that, just like $\mathrm{OC}, \mathrm{PP}$ cannot take into account the intuition that prescribing $\operatorname{drug} A$ is all-things-considered right and prescribing drug $B$ or drug $C$ is all-things-considered wrong. Table 3 shows what PP implies with respect to the Jackson case. ${ }^{5}$

PP entails that prescribing $\operatorname{drug} A$ is not right at all with respect to risk because there is no possible state in which prescribing $\operatorname{drug} A$ would have the best consequences. Prescribing drug $B(C)$ is right with respect to risk according to PP to the degree 0.5 because the state in which prescribing drug $B(C)$ has the best consequences $-s(t)$ - has a probability of 0.5 . Hence, just like OC, PP does not judge administering the perfect but very dangerous drug $(B$ or $C)$ allthings-considered wrong (as Jackson suggests). Moreover, like OC, PP does not take prescribing drug $A$ to be all-things-considered right. Rather, PP implies that prescribing $\operatorname{drug} A$ is entirely wrong with respect to risk.

To make things even worse for PP, the arguably most promising strategy for OC to deal with the Jackson case is not available to PP. Objective consequentialists can try to show that, while Jill ought to give the drug that completely heals John, her decision procedure should lead her to give drug $A$. (Smith 2006, Feldman 2012) The idea is that decision procedures, but not criteria of rightness, should take into account risk in a way that licenses Jill's prescribing drug $A$.

The decision-procedure solution, however, is not available to Peterson. As we discuss in more detail in section 3, Peterson recommends a decision method according to which agents ought to randomise in such a way that the 'moral force' of each option determines the probability with which the option ought to be performed. (117-121) The moral force of an option is the sum of the degree-strength products corresponding to each aspect where 'moral strength' is understood, roughly, as "the amount of moral value at stake" (37). In the Jackson case, prescribing $\operatorname{drug} A$ is not right at all and has, hence, zero moral force and zero probability of being performed according to Peterson's decision procedure. Thus, arguably unlike OC, PP is incompatible with a decision procedure that leads Jill to give $\operatorname{drug} A$.

\footnotetext{
${ }^{5}$ This agrees with Peterson's own MDC-based analysis of the case on page 107 (except that what is drug $A$ in Jackson's original example becomes drug $B$ in his own description).
} 
Table 3 MDC and the Jackson case

\begin{tabular}{llll}
\hline & State $s(p=0.5)$ & State $t(p=0.5)$ & Degree of rightness with respect to risk \\
\hline Drug $A$ & Partial cure & Partial cure & 0 \\
Drug $B$ & Death & Complete healing & 0.5 \\
Drug $C$ & Complete healing & Death & 0.5 \\
\hline
\end{tabular}

\section{b) Action-Guidance}

Objective consequentialism is often criticized for failing to be action guiding on grounds that we lack information about the consequences of our actions. ${ }^{6}$ Since you do not know the actual consequences of the acts available to you (after all, you would have to take into account all consequences until the end of days of any of the countless acts available to you), you do not know which of them are right and which are wrong. And therefore you cannot base your decisions on objective consequentialism's criterion of rightness, i.e., you cannot choose an act that is right according to objective consequentialism. So essentially, the problem is that you do not know how to decide on objective consequentialism.

Now, why is this a problem for PP? After all, if risk is admitted as a moral dimension, one might think, then all we have to know in those cases when we are not certain about the actual outcomes are the risk factors. However, this reply misunderstands the overall structure of MDC. We saw how this is supposed to work: we have to establish what each dimension contributes to the final all-things-considered moral verdict. Now, risk is only one dimension among others, and the other dimensions are understood along OC lines: apart from the risk dimension, the final verdicts about acts are based on actual consequences with respect to wellbeing and equality.

\section{c) 'Ought' Implies 'can'}

According to an influential argument, put forward most forcefully by Frances Howard-Snyder (1997), OC is false because it violates the principle that 'ought' implies 'can', i.e., the thesis that a person is morally obligated to $\varphi$ only if the person can $\varphi .{ }^{7}$ Howard-Snyder argues for the incompatibility of OC and the 'ought' implies 'can' principle by means of 'the Karpov case' (Ib.: 242): something important (say, a career) hangs on a mediocre chess player winning a chess championship by defeating Karpov. The mediocre player's attempt fails.

The crucial feature of the case is that the chess player could not have beaten Karpov and therefore could not have produced the best consequences. Since, according to OC, she ought to have done so (by assumption something crucial depends on it), we seem to get a violation of the 'ought' implies 'can' principle. As explained above, according to PP, apart from the risk dimension, actual consequences (regarding well-being and equality) affect the rightness or wrongness of actions. In the Karpov Case your well-being depends on the outcome of the match, and if $\mathrm{OC}$ is false because it is incompatible with the 'ought' implies 'can' principle, then so is PP. ${ }^{8}$

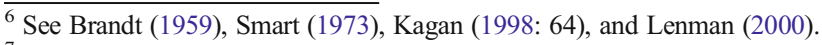

${ }^{7}$ See also McCloskey (1973).

${ }^{8}$ For an attempt to show that OC is compatible with the 'ought' implies 'can' principle, see Carlson (1999) and Andrić (forthcoming).
} 


\section{d) Decisiveness of Optimal Consequences}

In his discussion of the Jackson case, Peterson emphasizes that PP, in contrast to SC, preserves the intuition that "only optimal outcomes contribute towards making right acts right" (108). Peterson thinks that this preservation is an advantage of OC and PP - and the lack thereof correspondingly poses a problem for SC.

However, it is not true that PP preserves the intuition that only optimal outcomes make right acts right. For on PP the reduction of risk also contributes towards making right acts right. Return to the Jackson case with some modification. Suppose that Jill's evidential situation changes so that prescribing drug $B$ will lead to a complete cure with a probability of $90 \%$. Then the degree of rightness of prescribing drug $B$ will be 0.9 . Rightness has been increased by the reduction of risk. In sum, if a consequentialist doctrine should better accommodate the intuition that only optimal outcomes make right acts right, then there is not only a problem for SC but also for PP.

\section{e) Normative Disagreement}

Another possible problem for SC is its inability to make sense of normative disagreement. (Kolodny and MacFarlane 2010) To illustrate, here is the problem applied to the Jackson case. Imagine the following conversation:

Jill: I ought to prescribe $\operatorname{drug} A$, guaranteeing partial cure without the risk of death. Adviser: No, you ought to prescribe drug $B$. Doing so will completely heal John.

Assume that Adviser knows that drug $B$ will completely heal John. On SC, Adviser points out that prescribing drug $B$ has most expected value - from Jill's perspective. But this is not what is going on. The 'ought' of the advice is apparently an objective, perspective-independent 'ought' and SC cannot make sense of this.

A proponent of PP can indeed make better sense of the advice in the Jackson case than a proponent of SC: Although prescribing drug $B$ is wrong to a certain degree with respect to the risk dimension, it is completely right with respect to the dimension of well-being. This tends to make prescribing drug $B$ all-things-considered right to a high degree on a charitable reading of what the outcome of aggregation would be. However, this is not the end of the matter. The trouble for Peterson is that the problem reappears in cases where risk (instead of well-being) is the dominant aspect when it comes to the all-things-considered moral judgement. Here is one such case:

\section{BMWs or Torture.}

Alice can choose between three doors. All the evidence suggests, as is in fact the case, that she will be gifted six BMWs behind door no. 1 and tortured behind door no. 3. The evidence also suggests that Alice will almost certainly be tortured behind door no. 2, but there is a very small chance that she will be gifted seven BMWs. In fact, however, door no. 2 yields seven BMWs.

On PP, it is almost entirely right for Alice to choose door no. 1 with respect to both risk and well-being. Choosing door no. 2 is almost completely wrong with respect to risk and completely right with respect to well-being. Choosing door no. 3 is completely wrong. Assuming this analysis is correct, we cannot make sense of the following dialogue on PP: 
Alice: I ought to choose door no. 1 because I will get six BMWs this way and do not run the risk of being tortured.

Adviser: No, you ought to choose door no. 2. For then you will be gifted seven BMWs.

The 'ought' of the Adviser this time spells trouble for a proponent of PP: since the risk is extremely high that behind door no. 2 Alice will receive nothing but torture and all she would win is one more BMW in comparison to door no. 1 - which would yield more than enough BMWs -, it is almost entirely all-things-considered wrong for her to choose door no. 2. ${ }^{9}$ The bottom line is that if there is a problem for proponents of SC to make sense of the 'oughts' of better informed advisers, then PP (sometimes) suffers from the same problem.

\subsection{The problems of MDC}

In the previous section we have substantiated our claim that all problems of OC and SC are also problems for PP. One could object that, in order to assess whether MDC can handle risk better than one-dimensional consequentialist theories, one should look at the best version of MDC. And this may be different from PP. Our response is twofold. First, even if there were a version of MDC that, unlike PP, is superior to OC and SC when it comes to taking into account risk, the result that PP shares all problems of OC and SC would still be interesting. After all, PP, up to this point, is the only version of MDC that has been formulated. Secondly, however, it is in fact not the case that MDC would be superior to OC and SC on any version. So abandoning PP in favour of another version does not get MDC off the hook, as we want to show in this section.

What, if not PP, could be the best version of MDC when it comes to taking risk into account? We see three strategies for improvement. The first is to render MDC completely objectivist or completely subjectivist to avoid either all objections against SC or all objections against OC, respectively. While PP is a mix of subjectivism in the risk dimension and objectivism with respect to equality and well-being, the new version would either simply delete the dimension of risk and stick to the objectivist dimensions of equality and well-being or it would "subjectivise" the dimensions of equality and well-being by taking into account probabilities (probably accompanied by a deletion of the dimension of risk, which would then seem to be superfluous).

Admittedly, this manoeuvre would solve one kind of problem. However, on the new version of MDC, risk would no longer be one of several dimensions but either determine the deontic status of acts completely or not at all. But then MDC would simply become a multi-dimensional version of either OC or SC. In short, MDC would collapse into either OC or $\mathrm{SC}$. This cannot be a way out for someone who claims that MDC is superior to OC and SC when it comes to handling risk. For the new version would share all the problems of either OC or SC.

The second idea is to formulate a version of MDC according to which expected value, rather than best possible consequences (as in PP), determines rightness with respect to risk. Arguably, this version of MDC could handle the Jackson case, because it would judge prescribing drug $A$ right and prescribing drug $B$ or $C$ wrong with respect to risk (though, it

\footnotetext{
${ }^{9}$ If the difference between doors no. 1 and 2 in terms of well-being is not sufficient to make choosing door no. 1 almost entirely right according to PP (with respect to well-being and, in consequence, all-things-considered), one can amplify the marginal utility, for example by letting door no. 1 yield 1,000,000,000 ice-cream cones and door no. 2 1,000,000,001 ice-cream cones.
} 
would still contradict the intuition that prescribing $\operatorname{drug} A$ is all-things-considered right and prescribing drug $B$ or $C$ all-things-considered wrong). However, all the other problems we have discussed remain. Hence, again, this appears to be no solution for the proponent of MDC.

Finally, recall that we have mentioned in section 2.a) that some proponents of OC try to show that, on their theory, Jill's decision procedure should lead her to give $\operatorname{drug} A$ in the Jackson case. We have explained that the decision-procedure solution does not work for PP because Peterson recommends a decision method according to which agents ought to randomise whereby the probability with which the option ought to be performed is the sum of the degree-strength products corresponding to each aspect, which is zero for prescribing $\operatorname{drug} A$. The idea now is that the proponent of MDC abandons Peterson's randomisation method of decision-making in favour of a decision procedure that licenses prescribing drug $A$.

There are, however, two serious obstacles to this solution. First, one might well wonder if there is any alternative to Peterson's randomisation procedure available at all. After all, Petersons considers and rejects several alternatives to his randomisation method that might be correct decision procedures for one-dimensional but not for multi-dimensional moral theories $(115-6,121-38)$. It would go beyond the scope of this paper to discuss these arguments. The point is that the proponent of MDC who wants to adopt an alternative to the randomisation procedure needs to present a decision procedure that withstands Peterson's arguments. At the same time, in order to handle the Jackson case, the new procedure needs to license Jill's prescribing $\operatorname{drug} A$. We do not claim that there is no such procedure. But the burden of proof is certainly on the proponent of MDC.

Furthermore, even if we assumed that there is a superior alternative to Peterson's randomisation procedure which does indeed license Jill's prescribing drug $A$ on MDC, this assumption would only show that the response some proponents of OC give to the Jackson case is also available to the advocate of MDC. It would not show that MDC is superior to OC and SC when it comes to taking into account risk. Far from it; MDC would still face all the other problems we have presented in section 2 .

We conclude that the third strategy might not be viable and, at best, would be of no real help for the proponent of MDC. We know of no other strategy to improve MDC's handling of riskat least of no strategy which would not also be open to one-dimensional consequentialists and thus be of help in the present discussion. Nor does there seem to be any promising combination of the presented strategies. Hence, the objection that by comparing PP to OC and SC we did not choose the best version of MDC fails.

\section{Conclusion}

To sum things up: We have argued that the major problems that haunt $\mathrm{OC}$ and SC will not disappear with PP. At best, they will occur less often. Moreover, we have argued that the possibilities of finding a version of MDC that is significantly superior to PP as far as risk is concerned are very limited. However, there is also a glimmer of hope: Showing that risk is only one among several dimensions would prove OC and SC wrong. Thus, despite having all (or at least many of the) problems of OC and SC, MDC could eventually turn out to be the best consequentialist theory about risk.

How could the multi-dimensional consequentialist show that risk is only one among several dimensions? It is a bad idea to point out general or structural problems of OC and SC since we have seen that MDC shares these problems. The way to go is, rather, bottom up: The multi- 
dimensional consequentialist should look for cases that convey that actual consequences and risk form (or belong to) distinct dimensions. Peterson has not yet tried this path, as far as we can see. ${ }^{10}$ Our advice to him and to friends of MDC in general is, therefore, to pursue this line of inquiry in the future.

Acknowledgments We would like to thank the participants of the workshop "The Dimensions of Consequentialism", which took place at Konstanz University in November 2013. We are also very grateful for the comments received from Martin van Hees, Sebastian Köhler, and an anonymous referee. This research was supported by a grant from the German Research Foundation (Grant number TA 820/1-1).

Open Access This article is distributed under the terms of the Creative Commons Attribution 4.0 International License (http://creativecommons.org/licenses/by/4.0/), which permits unrestricted use, distribution, and reproduction in any medium, provided you give appropriate credit to the original author(s) and the source, provide a link to the Creative Commons license, and indicate if changes were made.

\section{References}

Brandt R (1959) Ethical theory: the problems of normative and critical ethics. Prentice-Hall, Englewood Cliffs Carlson E (1999) The oughts and cans of objective consequentialism. Utilitas 11(1):91-96

Feldman F (2012) True and useful: on the structure of a two level normative theory. Utilitas 24(2):151-171

Howard-Snyder F (1997) The rejection of objective consequentialism. Utilitas 9(2):241-248

Jackson F (1991) Decision theoretic consequentialism and the nearest and dearest objection. Ethics 101:461-482 Kagan S (1998) Normative ethics. Westview Press, Boulder, Co.

Kolodny N, MacFarlane J (2010) Ifs and oughts. J Philos 107:115-143

Lenman J (2000) Consequentialism and cluelessness. Philos Public Affairs 29(4):342-370

McCloskey HJ (1973) Utilitarianism: two difficulties. Philos Stud 24:62-63

Peterson M (2013) The dimensions of consequentialism. Cambridge University Press, Cambridge

Smart JJC (1973) An outline of a system of utilitarian ethics. In smart J J C and Williams B. Cambridge University Press, Cambridge, Utilitarianism - for and against

Smith M (2006) Moore on the right, the good, and uncertainty. In: Horgan T, Timmons M (eds) Metaethics after Moore. Clarendon Press, Oxford, pp. 133-148

\footnotetext{
${ }^{10} \mathrm{He}$ has presented cases that are supposed to suggest that there are several moral dimensions $(2,11,13,49)$, but he has not presented cases that suggest that risk is a separate dimension.
} 